\title{
Drowning in data: early responses of renal cortical collecting duct cells to lithium
}

\author{
John Leader, Jennifer Bedford, Robert J. Walker \\ Department of Medicine, University of Otago, Dunedin, New Zealand \\ Correspondence to: Prof. Robert J. Walker. Department of Medicine, University of Otago, Great King Street, Dunedin 9054, New Zealand. \\ Email: rob.walker@otago.ac.nz. \\ Provenance: This is an invited article commissioned by the Section Editor Dr. Linpei Jia (Department of Nephrology, Xuanwu Hospital of Capital \\ Medical University, Beijing, China). \\ Comment on: Sung CC, Chen L, Limbutara K, et al. RNA-Seq and protein mass spectrometry in microdissected kidney tubules reveal signaling \\ processes initiating lithium-induced nephrogenic diabetes insipidus. Kidney Int 2019;96:363-77.
}

Submitted Sep 03, 2019. Accepted for publication Sep 20, 2019.

doi: 10.21037/atm.2019.09.120

View this article at: http://dx.doi.org/10.21037/atm.2019.09.120

It is now more than fifty years since Burg made a signal advance in the study of renal physiology by reporting a method of isolating and perfusing tubules from selected regions of the kidney (1). Prior to this, knowledge of renal tubular function had been derived either from micropuncture experiments, limited to the superficial regions of the kidney (2), from kidney slices (3-6), which were subject to injury and represented an aggregate of tubular function, or from renal cells in tissue culture (7-9).

Since the early 1980's the rapid growth of methods for the analysis of the composition of individual tubular cells has seen a rapid increase in genomic information. This has been confused in the past by inconsistent terminology. Critical to the proper assignment of genomic data is to ensure that such data is correctly mapped to a particular cell type. In an important paper dealing with this (10), Knepper and co-workers proposed a nomenclature for tubular cell types, based upon the occurrence of cell type selective markers, characterised by high concentration of a specific protein, and consistent with assignations from the pregenomic era. However, until recently progress in studying the genome of a particular cell type has been limited by the small size of particular segments, typically only a few millimetres in length and containing only a few cells. It is only recently that new analytical methods, single cell RNA-Seq based transcriptomics, and LC MS/MS have allowed the determination of processes in individual cell types.

Knepper and colleagues have applied these techniques to a well-studied phenomenon, the effect of lithium on the renal tubules (11). It has been known for many years treatment with the lithium chloride, an effective treatment for manic depression, can lead to a loss of urinary concentrating ability (12), and ultimately over time, chronic kidney disease manifested as a chronic interstitial fibrosis $(13,14)$. Early physiological studies showed that lithium enters principal cells of the collecting duct, through the sodium channel $\mathrm{ENaC}$, with increased intracellular concentrations since the exit transport mechanisms are poorly selective for lithium. Intracellular lithium has been shown at a physiological level to inhibit both the synthesis of the water channel Aquaporin2, and its translocation to the apical membranes of principal cells (12). Lowered permeability of these membranes to water leads to polyuria. Knepper and colleagues (11) have used the techniques of RNA-Seq transcriptomics to characterise the response to short-term (up to 72 hours) application of lithium to microdissected regions of the collecting duct of rats, and LC-MS/MS to determine proteomic changes over the same time course, to investigate the initial signalling events.

It is well known that in animal models, lithium chloride treatment over four weeks results in proliferation of collecting duct principal cells and a subsequent increase in intercalated cells (15). To further investigate the time course of this process, immunohistochemistry using antibodies to AQP2 to identify principal cells, and to $\mathrm{H}+/$ ATPase to locate intercalated cells in microdissected tubules, found that there was a small increase in the 
number of AQP2 positive cells after 72 hours but no change in $\mathrm{H}+$ /ATPase positive cells, although there was a variable number (up to $15 \%$ ) of hybrid cells expressing both proteins. Knepper et al. (11) demonstrated that of the 6978 transcripts identified in the LiCl-treated cortical collecting ducts (CCD), 728 were increased compared with the TAL, and 370 were decreased. This was compared to the RNA-Seq of microdissected thick ascending limbs (TAL) which only showed 18 transcripts out of 6,194 to have a significant change in response to lithium exposure as compared to controls. In the CCDs, those transcripts which were decreased with lithium treatment had low $\mathrm{P}$ values and were not informative. However, those transcripts which were increased with lithium exposure were of considerable interest. The transcripts fell into several groups. Prominent were transcripts of protein kinases involved in cell cycle control, supporting the earlier conclusion of Christensen et al. (15) that the initial response of principal cells to lithium entry is activation of a proliferative response. Additionally there was an increase in transcripts from genes coding for a variety of chemokines associated with NF- $\mathrm{\kappa B}$ activation, p53 signalling, Wnt signalling and Aldosterone upregulated genes (11). Of particular interest was the abundance of several Wnt- $\beta$ catenin transcriptomes (11), since it is well known that nuclear $\beta$-catenin abundance is regulated by GSK-3 $\beta$, itself a target for lithium and a regulator of many key intracellular pathways. Of the key targets of Wnt- $\beta$ catenin, cyclin A2 was markedly increased and cyclin A2 activates critical kinases in cell cycle regulation as mentioned above (11).

A further study of the time course of activation of the early response genes revealed a rapid rise, over 24 hours, in abundance of transcripts of known immediate early genes, followed by a decrease, whereas gene groups associated with a proliferative response showed little activity at 24 hours but a progressive increase thereafter. In contrast there was a maintained decrease in transcripts encoding for Transporters and Channels, consistent with the observed physiological responses (11). Interestingly, use of the antiinflammatory agent dexamethasone, which blocks the inflammatory response to an increase in NF- $\kappa \mathrm{B}$, resulted in an increase in AQP2 abundance in Li-treated rats (11). It would have been of interest, to know what if any impact dexamethasone had on the other up-regulated pathways.

The study was extended to investigate protein abundance in lithium-treated rats to detect whether it was possible to identify the same processes as found with RNA-Seq, taking advantage of recent improvements in sensitivity of Mass Spectrometry. From rats treated with lithium for 72 hours a total of 2,469 proteins were detected, of which 1,057 could be quantified. Of these a total of 73 proteins showed significant change in abundance. Three of the four proteins which showed the greatest increase were associated with cell-cycle proteins, including PCNA, STMN1 and ERH, all of which has revealed increases in mRNA abundance. In marked contrast, only one AQP2 peptide was successfully quantified (11).

This paper is a tour-de-force, providing a vast amount of dynamic molecular data which offers highly significant opportunities as a scaffolding for further studies. The authors are to be highly commended for the rigour of their experimental work and subsequent analysis. Astonishingly, the huge perturbations resulting from the entry of lithium into the cells of the CCD represent the coordinated response of many genes associated with several different pathways, the overall significance of which remain to be elucidated. This further reinforces a recent editorial by Kishore and Ecelbarger (16) who suggested that lithium has become a versatile investigative tool in furthering our understanding of the molecular physiology of the kidney. These findings are challenging some long-held views in renal physiology, and presenting paradoxical changes in renal pathophysiology related to acute and long-term exposure to lithium. The power of modern bioinformatics and increasing knowledge of the pathways involved, serve to illustrate the complexity of living things.

\section{Acknowledgments}

None.

\section{Footnote}

Conflicts of Interest: The authors have no conflicts of interest to declare.

Ethical Statement: The authors are accountable for all aspects of the work in ensuring that questions related to the accuracy or integrity of any part of the work are appropriately investigated and resolved.

\section{References}

1. Burg M, Grantham J, Abramow M, et al. Preparation and study of fragments of single rabbit nephrons. Am J Physiol 
1966;210:1293-8.

2. Windhager EE, Giebisch G. Micropuncture study of renal tubular transfer of sodium chloride in the rat. Am J Physiol 1961;200:581-90.

3. Bray GA. Freezing-point depression of rat kidney slices during water diuresis and antidiuresis. J Physiol 1960;199:915-8.

4. Wedeen RP, Weiner B. The distribution of p-aminohippuric acid in rat kidney slices. Kidney Int 1973;3:205-13.

5. Macknight AD, Pilgrim JP, Robinson BA. The regulation of cellular volume in liver slices. J Physiol 1974;238:279-94.

6. Arakawa H, Washio I, Matsuoka N, et al. Usefulness of kidney slices for functional analysis of apical reabsorptive transporters. Sci Rep 2017;7:12814.

7. Hopps HE, Bernheim BC, Nisalak A, et al. Biologic characteristics of a continuous kidney cell line derived from the African Green Monkey. J Immunol 1963;91:416-24.

8. Detrisac CJ, Sens MA, Garvin AJ, et al. Tissue culture of human kidney epithelial cells of proximal tubule origin. Kidney Int 1984;25:383-90.

9. Kreisberg JI, Wilson PD. Renal cell culture. J Electron Microsc Tech 1988;9:235-63.

Cite this article as: Leader J, Bedford J, Walker RJ. Drowning in data: early responses of renal cortical collecting duct cells to lithium. Ann Transl Med 2019;7(Suppl 8):S332. doi: 10.21037/ atm.2019.09.120
10. Chen L, Clark JZ, Nelson JW, et al. Renal-tubule epithelial cell nomenclature for Single-Cell RNAsequencing studies. J Am Soc Nephrol 2019;30:1358-64.

11. Sung CC, Chen L, Limbutara K, et al. RNA-Seq and Protein Mass Spectrometry in microdissected kidney tubules reveal signalling processes that initiate lithiuminduced Diabetes Insipidus. Kidney Int 2019;96:363-77.

12. Marples D, Christensen S, Christensen EI, et al. Lithium-induced downregulation of Aquaporin-2 water channel expression in rat kidney medulla. J Clin Invest 1995;95:1838-45.

13. Walker RJ, Leader JP, Bedford JJ, et al. Chronic interstitial fibrosis in the rat kidney induced by long-term (6 months) exposure to lithium. Am J Physiol Renal Physiol 2013;304:F300-7.

14. Presne C, Fakhouri F, Noel L, et al. Lithium-induced nephropathy: rate of progression and prognostic factors. Kidney Int 2003;64:585-92.

15. Christensen BM, Kim YH, Kwon TH, et al. Lithium treatment induces a marked proliferation of primarily principal cells in rat kidney medullary collecting duct. Am J Physiol Renal Physiol 2006;291:F39-48.

16. Kishore BK, Ecelbarger C. Lithium: a versatile tool for understanding renal physiology. Am J Physiol Renal Physiol 2013;304:F1139-49. 